\title{
Explosions, Burn Injuries and Adverse Health Effects of Electronic Nicotine Delivery Systems: A Review of Current Regulations and Future Perspectives
}

\author{
Ayesha Rahman Ahmed ${ }^{1}$, Beverly Etchey ${ }^{1}$, and Mahiba Ahmed ${ }^{2}$ \\ ${ }^{1}$ Department of Pharmaceutical Sciences, Washington State University, College of Pharmacy and Pharmaceutical Sciences, \\ Spokane, Washington, USA; ${ }^{2}$ Voiland School of Chemical Engineering and Bioengineering, Pullman, Washington, USA
}

Corresponding author: Ayesha Rahman Ahmed, Ph.D., Department of Pharmaceutical Sciences, Washington State University College of Pharmacy, SPBS Room 409, 412 E. Spokane Falls Blvd, Spokane, WA 99210, USA; TEL: (509)368-6667; email: ayesha.ahmed@wsu.edu

Received, August 5, 2021; Revised, August 30, 2021; Accepted August 30, 2021; Published, September 1, 2021

\begin{abstract}
Purpose: While there is a declining trend in the use of traditional methods of smoking tobacco, electronic nicotine delivery systems (ENDS) have gained popularity worldwide. ENDS are marketed as safe for the primary reason that they do not contain the well-established toxic ingredients found in traditional cigarettes. However, growing concerns over incidences of fire and explosion with specific types of ENDS, as well as their short and long-term effects, remain unaddressed. This review examines the under studied role of customized components such as batteries, e-liquid compositions, and methods of nicotine delivery that result in physical injuries and adverse health effects of ENDS. Methods: Using online reference databases (Web of Science, PubMed, Medline other, Google scholar, FDA website, FDA register), we analyzed the mechanisms through which ENDS may pose significant risk to human health. Results: An increase in the use and popularity of ENDS has been observed among youth and adults in the United States since 2007. The ENDS devices available to the public allow for custom alterations which can introduce incompatible components, resulting in overheating and explosion related injuries. Heavy metals have been found to leach from some devices into the e-liquid, and the heating of eliquid ingredients can produce toxic byproducts. Conclusions: Overall, the current literature demonstrates that ENDS are not a safe alternative to traditional cigarettes due to explosion risks and negative health effects including addiction, adverse respiratory and cardiovascular effects, heavy metal leaching, and toxic byproducts exposure. These risks warrant regulation of ENDS devices and formulations, with urgency underscored by their increasing popularity among youth and adults.
\end{abstract}

\section{INTRODUCTION}

Electronic cigarettes or electronic nicotine delivery system (ENDS) were first introduced to the United States in 2007 and marketed as safe for the primary reason that they did not contain the well-established toxic ingredients found in traditional cigarettes. Conceptually, ENDS are designed to resemble traditional cigarettes. They consist of an aerosol generator, flow sensor, battery, and cartridge for eliquid storage and are available in a range of designs including e-cigarettes, advanced personal vaporizers (APV) and personal vaporizers (PV). Materials used to make the ENDS device include metal, ceramics, plastic, foam and more. Current ENDS devices offer advanced user-modifiable features such as the selection of heating temperatures and the rate of air flow that can be powered by manufacturer supplied rechargeable batteries, regular batteries, or a USB power cord source (1).

A recent systemic review on the injuries related to ENDS overheating, ignition, and explosion has been reported (2). However, the regulatory aspect of ENDS and constituents of e-liquid used in ENDS in the United States and worldwide, short- or long-term impact on the healthcare system, and the potential risks associated with heavy metal leaching, toxicities caused by e-liquid by-products, and direct or secondhand pulmonary and cardiovascular toxicities remain to be addressed. The surge in manufacturing capacity, availability of ENDS, and increasing popularity among cigarette smokers have prompted growing safety concerns from the public, healthcare providers and regulatory authorities including the Food and Drug Administration (FDA). Current studies and literature demonstrate that ENDS use is not without risk. This review deciphers the role of 
batteries, e-liquids, nicotine delivery, and unique health challenges that ENDS pose due to the accidental risk of explosions and burn injuries, potential addiction, and adverse health effects that provides rationale for stricter regulation of ENDS products.

A detailed search of the literature was conducted from October 2011 to September 2020 to identify research related to e-cigarettes and/or ENDS. Reference databases (Web of Science, PubMed, Medline other, Google scholar, FDA website, FDA register) were searched using a set of relevant search terms or keywords in combination. Search terms included the following: "ENDS explosion," "ENDS injury," "ENDS fire," "ENDS hazard," "ENDS battery explosion," and "ENDS health effects."

The articles and case reports were assimilated and reviewed if they were written in English, freefull text available, and dealt partly or exclusively with ENDS explosions or fires, health effects and eliquid toxicity. Articles were excluded if they were only focused on traditional cigarettes or health effects not associated with ENDS, abstracts, or documents from conference presentations/posters, not published in peer-reviewed journals, national and international standards, and government reports.

A total of 120 articles were identified for review. Article titles and abstracts were screened by the authors for applicability. There was no utilization of a conflict resolution process. The free full-text articles were manually selected and reviewed for relevance. Following the review, 84 full-text articles were found to be relevant for analysis. The validity and strength of each study were determined based on a qualitative assessment of the objectives and findings of the study. Structure of the proposed framework for the review is provided in the flowchart (Figure 1).

The following sections of the review provide a detailed analysis of ENDS and their negative health impact in ways that are similar or contrasting to the health hazards from traditional cigarette smoking.

\section{BATTERIES: EXPLOSIONS, FIRES AND BURNS}

Due to the dearth of the current literature on the mechanical safety of ENDS devices, there is a paramount need to determine the ENDS battery composition and factors that contribute to the risk of overheating, fires, or explosions due to battery failures. The proposed phenomenon of "thermal runaway," in which the battery overheats and causes a fire or explosion, is increasingly reported by ENDS users $(3,4)$. The new designs of ENDS allow custom alterations of the device by the user, including different options for cartridges and heating temperatures of the atomizer. However, incompatibility among the components can lead to overheating of the device. Factors that potentially contribute to ENDS overheating, fire and explosion include: the integrated safety circuit design, custom device modification by the ENDS user, incompatible or poorly designed devices, the types and quality of batteries or chargers, and the lack of quality control by suppliers (5). Favorability in the use of lithium batteries as the power-source in ENDS stems from their compact size and long-lasting power supply. However, studies point toward "thermal runaway" associated with these batteries as a cause for concern in the safety of ENDS $(1,6)$.

To highlight the potential hazards associated with incompatibility between the ENDS device and battery, a report noted that 15 patients were treated for injuries incurred from the explosions of ENDS lithium-ion battery component between October 2015 through June 2016. The injuries included flame burns, chemical burns, and blast injuries to the face, hands and thigh or groin. The injuries described in the report resulted in tooth loss, extensive loss of soft tissue and other physical injuries (3). Similarly, an attempt to improve the vaping experience by modifying the heating element subjected an individual to severe ocular injuries from an explosion. The different types of ocular trauma associated with ENDS injuries include thermal, contusional, penetrating and chemical injury, most likely originating from the alkaline battery of the device (7). According to a report, the explosions from the ENDS were initially thought to be rare, but there were published reports that showed 25 separate incidents of e-cigarette explosions from 2009 through 2014 across the United States. National Electronic Injury Surveillance System (NEISS) reported approximately 75 cases from 2015 until December 2019, with battery explosion in pants pockets leading to injury on thigh, calf, abdomen, buttock, hand, groin area etc. (8). Although burn injuries from ENDS are not common, the force of the explosion of the battery body leads to injury patterns that require treatment in specialized centers with the entire spectrum of burn care. Mixed injuries with deep burns occur due to thermal action, colliquative necrosis due to leaking electrolyte fluid and 


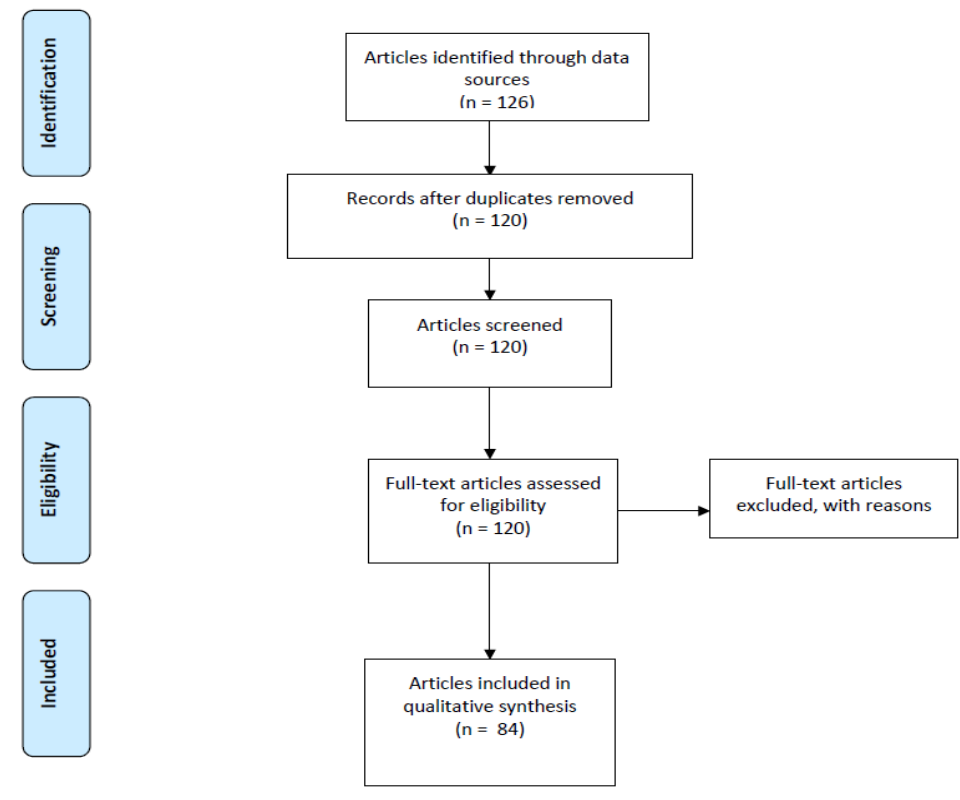

Figure 1. Structure of the proposed framework for the systematic review is provided in the flowchart.

contamination by foreign bodies (metallic lithium, splinters of the destroyed housing). The extinction of fire caused by e-cigarettes also requires special measures. Proper handling of Conformité Européenne (CE)-certified devices is essential because any manipulation of the device in the event of battery or functional defects is highly dangerous. Therefore, security precautions must be observed (8).

Though many reported ENDS explosions occurred independent of the use of the device, injurious explosions have occurred during device operation (9). Injuries resulting from such operational failure include trauma to the oral cavity, leading to possible complications via aspiration of the broken device parts. One case report described injuries sustained by a patient from the explosion of his ENDS, including oral burns, lacerations, and tooth fractures, as shown in Figure 2 (10).

Another case report described extensive burns that were sustained by a patient following the explosion of ENDS device. Due to the patient mixing the "e-liquid" with water, no toxic effect was observed in this case (11). The dilution of e-liquid with water result in a reduction in the overall physical boiling point of the solution, thus the potential scald injuries of the e-liquid on the burned skin were not observed in this patient case (11). Burns to the thigh were described in two case studies which led to the conclusion that there is a risk of irritation to the injury site from water irrigation, possibly due to a reaction between the irrigation water and lithium ions from the battery explosion $(12,13)$.
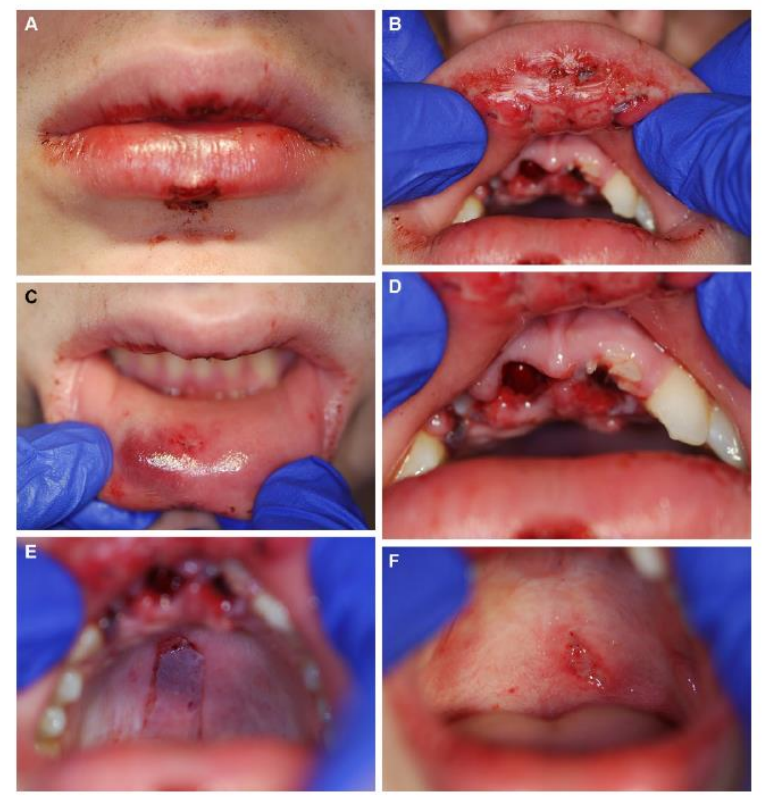

Figure 2. Examples of burn injuries and facial tissue damage. A) Extraoral view of lips. B) Upper labial mucosa. C) Lower labial mucosa. D) Teeth involved. E) Hard palate. F, Soft palate [Adapted from Ref (10) with permission].

A retrospective case review of eight patients with ENDS-use related burn injuries led the authors to recommend the initial assessment of injuries based 
on the Advanced Trauma Life Support guidelines, serum heavy metal levels of lithium, cobalt, and manganese to be checked and monitored for metal toxicity, and wound site debrided and irrigated to remove any residual materials (14). A unique case was also presented where the explosion of an ENDS device dislodged the mouthpiece and fractured the $\mathrm{C} 1$ vertebrae in the neck along with burns to the patient's lips and tongue. The possibility that the foreign object in the neck could have caused damage to arteries and other important musculoskeletal structure poses an additional complication to be assessed by medical professionals while examining these types of injuries (15).

There are few case study reports of the projectile damage to the face resulting in corneal laceration, dental trauma, and toxic and/or caustic burns of the patients' ocular surface (16).

These injuries were attributed to the possible defective or incompatible electric currents from the batteries leading to thermal runaway, which results in an uncontrolled increase in temperature causing combustion (16). Another report described an uncommon high-pressure injection injury, wherein the ENDS explosion resulted in the injection of eliquid into the index finger of a healthy adult man. This type of injury is usually seen in industrial accidents, so the extent of the damage was not evident until radiographs of the hand were obtained showing the radiopaque e-liquid (17). With the increased use of ENDS and higher rates of explosion injuries, it was recommended that the management of injuries sustained from ENDS explosions should be performed based on the different mechanisms, which include thermal burns with flames (type A), blast lesions (type B), chemical alkali burns (type C), and thermal burn without flames (type D) (18). Patterson et al proposed a dual classification of burn injuries from ENDS explosions: direct/indirect and an additional arithmetic classification in types $1-5 \mathrm{~b}$ : types 1,2 , and 3 were defined by the body area affected (hand, face, waist, and groin), while type 5 included inhalation injuries from device on fire further subclassified in-5a (upper airway injuries from direct flash or explosion of the e-cigarette), and $5 \mathrm{~b}$ (chemical, subglottic smoke inhalation injury) $(19,20)$. Types $1,2,3$, and 5 a were the direct injuries. However, Type 4 was classified as indirect injury (house fire injury). The severity of burn injuries that can result from ENDS explosion was described in a recent case series, as shown in Figure 3 (21). The mechanism of explosion was explained as thermal runaway of the lithium battery while in use, stored in a pocket or charging, as shown in Figure 4 (21).

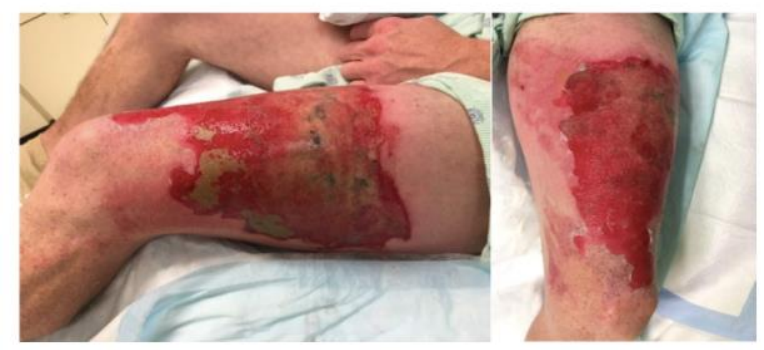

Figure 3. Elicited burn injuries from ENDS upon admission [Adapted from Reference (20) with permission].

The majority of ENDS-related traumatic, chemical, and thermal injuries were caused by the device and/or battery self-exploding. Explosion of the assembled device or battery when stored in the user's pants pocket explains why the majority of cases were men with thigh and or hand injuries. Warm and humid conditions and the presence of metallic objects such as keys have been proposed as possible causes of in-pocket explosions. The batteries used in ENDS have become more sophisticated and powerful over time, allowing users to alter power delivered to the coils and customize the vaping experience. Larger batteries allow for increased power delivery and extended usage time, but increased risk of serious injury. Thermal burns, alkali burns (from lithium hydroxide), house fires, and fatalities have been reported from ENDS use. Battery malfunction has been attributed to several factors, including faulty casing, over-heating, overcharging, exposure to perspiration (e.g., from pant pockets), or short-circuit by metal contact (e.g., with coins or keys) (22).

The failure rate of lithium batteries in relation to available e-cigarette devices is unknown. Fiery or explosive failures of lithium-ion batteries are reported to be rare and have occurred in cellphones, laptops, and other electronic devices. The element that distinguishes e-cigarettes from other devices is its cylindrical shape, while cellphones utilize a pouch-type battery and laptop batteries are contained in plastic cases. If the battery seal becomes damaged, the pressure within the e-cigarette increases to the point of explosion. According to a report published by the U.S. Fire Administration, 195 e-cigarette fires and explosion incidents attributed to the lithium-ion battery failure were described in the media from 2009 to 2016. It was explained that limited data are available since a majority of e-cigarette fires are self- 
or easily extinguished, so few were recorded in the

NFIRS database. The report advised consumers to

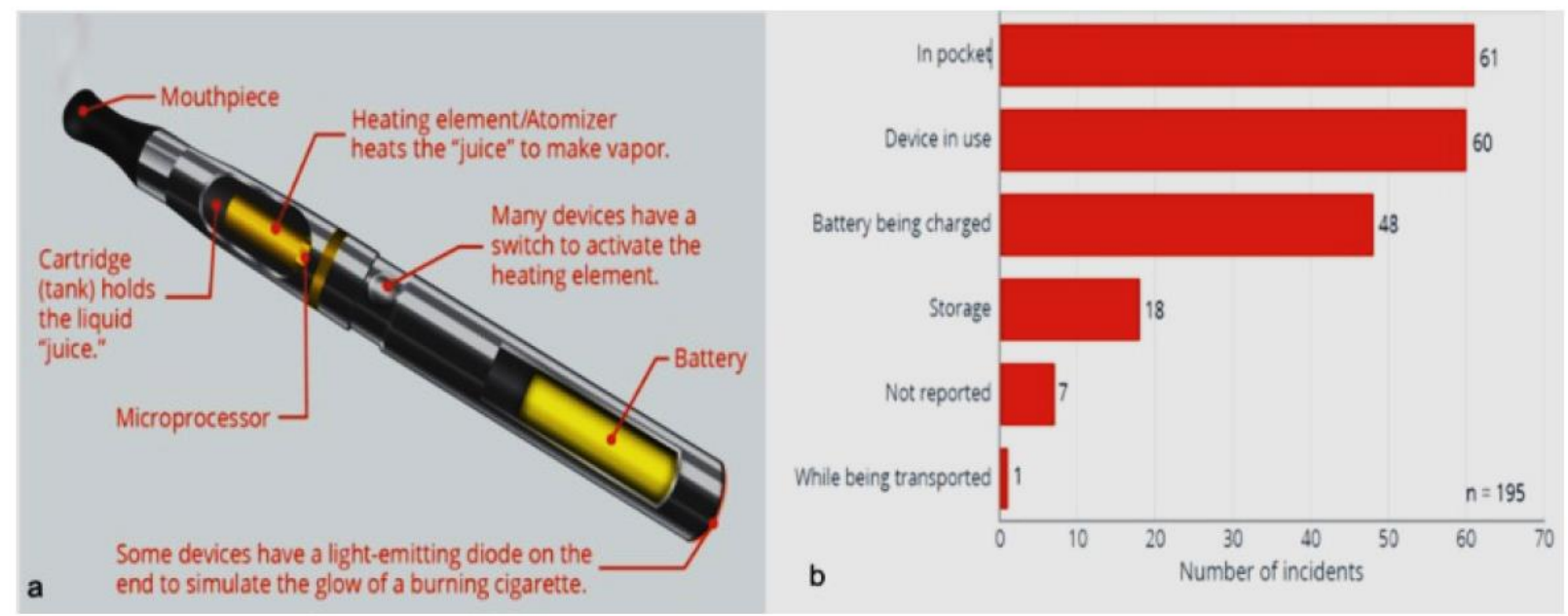

Figure 4. Parts of an electronic cigarette (a) and statuses of ENDSs when the incident occurred (b) [Adapted from Reference (21) with permission].

purchase UL safety standard batteries and potentially avoid lithium-ion battery for use in e-cigarette devices (23).

The increasing rise and popularity of ENDS has brought a surge of unregulated manufacturers and distributers of the ENDSs. Due to the present dangers of using ENDS, it is recommended that stricter regulations be made by the FDA for better manufacturing practices and restrictions on customization. Several countries have placed strict regulations on the use of ENDS as medical devices which require a prescription (24). It may not be feasible for the U.S. to implement such regulations currently, but it could be beneficial to enact additional restrictions such as requiring approval from health authorities on the components and specifications of ENDS.

The U.S. FDA published a final rule for the 2016 Amended Family Smoking Prevention and Tobacco Control Act to deem tobacco products, with the inclusion of ENDS products. Section 101 of the Tobacco Control Act amended the FD\&C Act with the addition of section 904 and 905. Section 905(b), "requires that every person who owns or operates any establishment in any State engaged in the manufacture, preparation, compounding, or processing of a tobacco product or tobacco product register with the FDA..." while section 904(a)(1) "requires each tobacco product manufacturer or importer to submit all ingredients that are added to the tobacco product, including ENDS" (25). It was stated that information will be collected from manufacturers to impose restrictions that will benefit and protect the public health. In deeming ENDS as a tobacco product, the FDA will be able to "review premarket applications for new tobacco products to increase product consistency" (25). This step by the FDA is commendable and will provide the data needed for stricter regulations and overall public health.

\section{E-LIQUID: COMPOSITION AND HEALTH EFFECTS}

An aspect of ENDS use that is currently being explored is the possibility that chemical alterations could produce short- and long-term health effects. The e-liquid contains chemicals that are mainly inert but could potentially generate harmful by-products when heated. These substances include nicotine, propylene glycol, glycerin, ethanol, flavoring agents, and contaminant heavy metals such as nickel, chromium, zinc, and silver which could leach from the device into the e-liquid (26). Propylene glycol and glycerin are two primary ingredients in e-liquid that function to blend the nicotine and flavoring agents, enhance absorption of the wicking material, and generate the aerosol when heated. Propylene glycol and glycerin are classified as safe for oral consumption by the FDA, however, the process of heating the compounds in an ENDS could be hazardous (27). Wang et al found that propylene glycol and glycerin produce toxic by-products such as formaldehyde, acetaldehyde and acrolein when 
heated in the e-liquid, leading to formaldehyde levels that exceed the acceptable daily limits set by the United States Environmental Protection Agency (USEPA) and California Office of Environmental Health Hazard Assessment (OEHHA) (28). Several other studies also suggest that the formaldehyde produced in dry puffs are much higher than that produced by cigarette smoke $(29,30)$. Another study suggested that propylene glycol and glycerin may be associated with ocular irritation, which was observed in 5\% of the cases associated with ENDS use (31). Regarding the flavoring agents, there is limited conclusive data on the health effects that could potentially arise from the inhalation of agents such as eugenol, benzaldehyde, and menthol (27). Recent studies point to the emerging evidence that cigarette menthol in may contribute to an increased risk for lung cancer by inhibiting the detoxification and clearance of the potent lung carcinogens $(32,33)$. Another study on the direct exposure of e-liquid containing coconut, vanilla and cookie flavors showed that mixed flavored e-liquid caused changes observed in long time cigarette smokers by inducing inflammation, cytotoxicity, and inhibiting wound healing responses in pulmonary fibroblasts (34). A recent study by Abouassali et al showed in vitro using HL-1 cardiomyocytes that e-vapors containing flavoring aldehydes such as vanillin and cinnamaldehyde can compromise cardiac electrophysiological functions, leading to action potential instability and inducible ventricular arrhythmias (35). Therefore, further studies are warranted for additional rigorous mechanistic insights for a better understanding of the unwanted effects of ENDS flavors.

The available evidence regarding health effects associated with short-term exposure to e-cigarettes was evaluated by the National Academies of Sciences, Engineering, and Medicine, Board on Population Health and Public health Practice committee. Overall, it was concluded that ecigarettes pose a potential health risk, and possibly less risk for users with existing respiratory disease, but the long-term effects on morbidity and mortality are unclear (36). The conclusions on health effects made by the committee were summarized based on the strength of evidence, whether, substantial, conclusive, moderate, limited, insufficient, or no available evidence. Of note, substantial evidence was found for heart rate increases after nicotine exposure from e-cigarettes, symptoms of dependence, formation of reactive oxygen species/oxidative stress, acute endothelial cell dysfunction and that some chemicals in e-cigarette aerosols (e.g., formaldehyde, acrolein) could cause DNA damage and mutagenesis (36).

Research on metal toxicity is currently emerging and has been proposed as a mechanism of injury from ENDS. The metals commonly found in the ENDS heating coils could potentially leach into the e-liquid and cause adverse effects. An analysis of ENDS refill bottles and cartridges from manufacturers including Joyetech, JUUL, Vuse, blu, Mistic and more, demonstrated the presence of metals such as $\mathrm{Cr}, \mathrm{Ni}, \mathrm{Cu}, \mathrm{Zn}, \mathrm{Cd}, \mathrm{Sn}$ and $\mathrm{Pd}$ (26). The metals were not only present in the liquid but also in the aerosol generated from heating the device. The variables found to influence the amount of metals in the aerosol included the changing levels of corrosion within individual devices, differences in diameters and compositions of heating elements, the power provided to the heating elements that produce the heat for liquid vaporization, and the differences in mouthpiece design that affect nebulization (26). A study of ENDS aerosol exposure to lung cells found high levels of copper nanoparticles which could contribute to oxidative stress and genotoxicity in lung cells. It was found that ENDS copper particles disrupted mitochondrial membrane potential, mitochondrial mass, ATP levels and mitochondrial reactive oxygen species in human airway epithelial cells (37). Aerosols generated from ENDS facilitate copper ion transport across plasma membrane, thereby posing a greater risk of free radicals mediated oxidative stress and DNA damage $(38,39)$. A recent study by Sharma et al showed that aerosols generated by nicotine-free e-cigarette use resulted in increased gut inflammation through barrier disruption of gut epithelial cells and downregulation of tight junction proteins in murine and human cells (40). The presence of toxic metals in an ENDS user was demonstrated in a toxicology report of a patient with a hand injury from an ENDS explosion. It showed an initial increase in cobalt and manganese in serum and a decrease in the metal concentration after treatment (24). Another study of ENDS users from Maryland indicated that metals in the aerosol, including $\mathrm{Ni}$ and $\mathrm{Cr}$, are inhaled, and absorbed into the body of the users (41). Although the systemic effects of the metals were not evident, there may still be many long-term negative health effects related to metal toxicity. The health effects of ENDS use are currently not well known or understood but should be considered by health professionals when caring for patients [16]. The findings of a case series by Kumetz et al suggested that physicians, especially 
those in emergency medicine, should be more aware of the hazards associated with ENDS use and educate patients when they present with injuries or adverse health effects based on the experiences of the patient cases, who had no knowledge of the risks of ENDS use prior to sustaining their injuries (42).

\section{NICOTINE: AN ADDICTION POTENTIAL AND ADVERSE HEALTH EFFECTS}

Nicotine is known to have both muscarinic (salivation, vomiting, diarrhea, and bronchial constriction) and nicotinic (hypertension, tachycardia, and muscle cramps) effects [30]. The use of and exposure to ENDS through inhalation is dramatically increasing among children and young adults. Though the reasons for ENDS initiation in young adults are not completely available, the unregulated marketing and promotion, provision of attractive flavourings, and the perception that ENDSs are "safe" compared to traditional cigarettes has led to the increased use of e-cigarettes in the youth (43). A study conducted in the U.K. involving the audit of different ENDS retailers found that the majority of customers were attracted to the affordability of ENDS either as a cessation aid or as a substitute for tobacco. The adolescent customers reported that they were mainly interested in the variety of flavours available, and perceived that the ENDS did not contain nicotine and were therefore safe for consumption (44). In addition, marked inaccuracies were detected in the nicotine concentration labelling of 12 e-liquids, which may further adversely affect the user habits and reliability of products used in smoking cessation (45). Mass marketing campaigns with the use of television and radio advertisements featuring celebrities, appealing flavours, and easy purchasing access through online retailers have led to the increased use of ENDS among children and adolescents (46). Concerns regarding ENDS use among children and young adults includes nicotine addiction and potential adverse effects on brain development, leading to poor academic achievement and cognitive problems (47).

Analysis of nicotine levels in various batches of electronic cigarette refill liquids indicated that more than $50 \%$ of the e-liquid samples had nicotine concentrations significantly above or below what is shown on the label. The actual variation ranged from $66 \%$ below the labeled amount to $172 \%$ more than the labeled amount (48). ENDS have been reported to increase heart rate and blood pressure, potentiating cardiac events and arrhythmia in people with or at risk of cardiac disease (49). A study among adolescents in Hong Kong found a strong association of respiratory symptoms with ENDS use in occasional ENDS smokers compared to the neversmoker population; a finding which was unlikely to be attributed to any confounding factors (50). Another study evaluating the oral health risks of ENDS use among adolescents found that ENDS use greatly increased the chance of cracked or broken teeth or tongue and/or inside-cheek pain, which was associated with the use of nicotine-containing ENDSs (51). Apart from the physiological effects of nicotine, it has been suggested that ENDS could potentially increase the odds among the adolescents for smoking conventional cigarettes (52). Since the long-term effects of ENDS are not currently known, it is important to educate parents, relatives and childcare providers on the importance of keeping the products out of reach of an adolescent population (53).

With some nuanced considerations, ENDS are expected to confer nicotine addiction. ENDS users may have been previously exposed to nicotine with traditional cigarettes or may be dual users of both traditional cigarettes and ENDS, which prompts the need for additional studies on addiction from ENDS alone. A case report described a patient with a 10year history of ENDS use alone, who presented to the emergency room with symptoms of diaphoresis, restlessness, tachycardia, chills, and nausea. The symptoms were found to be associated with abrupt discontinuation of ENDS and he was diagnosed with nicotine withdrawal (54). Nicotine dependence was also demonstrated in the adolescent ENDS users in a study that investigated cross-sectional data from the Texas Adolescent Tobacco and Marketing Surveillance System (TATAMS)(55). It showed that a significant number of adolescent sole ENDS users, 91 out of 132, reported symptoms of nicotine dependence, indicating that ENDSs can contribute to nicotine dependence among adolescents (55). Morean et al (56) performed the study on 520 adolescents and reported that ENDS could induce the nicotine dependence. Analysis of the nationally representative Population Assessment of Tobacco and Health (PATH) data set from 7025 adolescent participants suggested that parental and environmental factors altered the risk of initiation and persistence of ENDS usage, and should be critically considered when working with at-risk adolescent population (57). 
The nicotine dependence for the ENDS is based on vaping frequency, and with the exception of nicotine-free e-cigarette liquid, e-cigarette use can result in peak nicotine levels similar to combustible cigarettes and, therefore, could produce nicotine dependence over time $(58,59)$. ENDS liquid containing nicotine range from 9 to $24 \mathrm{mg} / \mathrm{mL}$. Nicotine delivery via tobacco smoke (cigarette) is absorbed within 10 to 20 seconds in high concentration through the lungs into the bloodstream, reaching the brain in the same high concentration within 10 seconds. Within 10 minutes of smoking, plasma nicotine peak at 15 to $30 \mathrm{ng} / \mathrm{mL}$. Only a few studies have explored plasma nicotine and plasma saliva cotinine levels via delivery from an e-cigarette. For studies on plasma concentrations of nicotine, the pattern is similar for the different studies with time points at $\mathrm{T} 0$ and 5 or 6 hour samples between 5 and 130 minutes after different "vaping" patterns: a fixed number of puffs or a fixed number plus a 60-minute ad lib period or a free number of puffs (60-66). Interindividual differences in cotinine levels could result due to several factors, some of which were measured in these studies (prior level of tobacco dependence, nicotine content in eliquids, number of puffs, and e-cigarette brand), and some which were not assessed, for example, electric power of the device, vapor temperature and density, nicotine concentration in the vapor (vs in liquids), volume of puffs, depth of inhalation, duration of apnea between inhalation and exhalation, and each individual's specific nicotine metabolism, but the major factor identified is the number of puffs (58).

The rate of nicotine dependence could vary by the nicotine content in conventional cigarettes. Nicotine delivery may be affected by smoking more cigarettes and/or smoking more intensely. The Fagerström Test for Nicotine Dependence (FTND) is a standard instrument for assessing an ordinal measure of nicotine dependence related to cigarette smoking. It evaluates the quantity of cigarette consumption, the compulsion to use, and dependence. Perkins et al (67) reported in 187 participants that 17 cigarettes per day scored 4.5 (medium dependence) in a scale of 0-10 on FTND. Another study by Bandiera et al [6] reported 20 cigarettes per day as the high score for FTND. The self-reported cigarettes per day (CPD) were categorized into "heavy" dependence and "hardcore" smokers for the study recruitment in different studies $(67,68)$. However, the strength of these associations and screening methods depended on the consistency and reliability of self-reported CPD, which remains unclear (69). Moreover, wide variability across days in cigarette intake may question the reliability of a single reported "usual" CPD and suggest the need for more careful, prospective assessments, both to detect such variability and to avoid under- or over-counting of the actual number of cigarettes consumed per day (67). Similarly, another commonly identified problem with smoker estimates of their "usual" number of CPD is "digit bias," in which their estimates are observed to be values that are even multiples of 5 cigarettes (70). Further research is needed to characterize the addictive and dependence potential of ENDS such as the nicotine concentration at which nicotine dependence can occur.

\section{IMPACT OF ENDS USE ON THE HEALTH CARE SYSTEM}

The use of ENDS not only impacts the health of the public, but also presents challenges to healthcare professionals. ENDS were introduced as a potential smoking cessation option due to their marketed ability to gradually decrease nicotine levels. Based on the previously reported evidence of the questionable safety of ENDS, it is important for healthcare professionals to educate and promote the smoking cessation that have been demonstrated to be effective and safe for patients, while discouraging the use of ENDS because of unknown long-term adverse health effects. FDA-approved nicotine replacement therapies and medications available to patients include nicotine patches, gums, inhalers, bupropion and varenicline (71).

Although the focus of this review is on the hazards of e-cigarettes, it is important to note that there are a number of studies on the potential use of nicotine-containing e-cigarettes for smoking cessation. A recent Cochrane review by HartmannBoyce presented findings that e-cigarettes with nicotine can assist with smoking cessation compared to e-cigarettes without nicotine and nicotine replacement therapies, though limitations included small sample of studies and short follow-up (72). Two studies by Polosa et al provided evidence that patients with asthma and COPD who switched from tobacco smoking to e-cigarettes experienced improvements in symptoms and pulmonary function for asthma patients as well as improved COPD exacerbation rates, which was predicted to persist long term $(73,74)$. The Vesuvius trial, a prospective, randomized controlled trial, studied the cardiovascular effects of switching from tobacco cigarettes to electronic cigarettes, with and without 
nicotine in 114 patients. With a primary efficacy endpoint of change in flow-mediated dilation, it was concluded that a switch from tobacco cigarettes to ecigarettes demonstrated benefits to vascular function. Of note, no difference was found in vascular benefit between e-cigarettes with nicotine and without nicotine and may require further study (75). In support, a randomized clinical trial of adults attending the U.K. National Health Service, randomized to either nicotine-replacement products of their choice (e.g., patch, gum, lozenge, nasal spray) or an e-cigarette starter pack, e-cigarettes were found to be more effective than nicotine-replacement therapy when both products are combined with behavioral support (76). The evidence for the use ecigarette as a smoking cessation aid may be valid, however, additional research on the efficacy and long-term safety are needed.

The concern for negative health effects is not only for the ENDS user but also for others who may be exposed through second-hand inhalation of vapor (77). Potential risks include respiratory and cardiovascular adverse effects as seen with direct use of ENDS, though not currently established for second-hand exposure. A policy statement from the American Association for Cancer Research and American Society of Clinical Oncology stated that risk from second-hand exposure to ENDS is possible, though there is no established data on potential health effects through this route. It was stated that some studies reported ultrafine particles, trace amounts of carbonyls, volatile organic compounds, polyaromatic hydrocarbons, tobacco-specific nitrosamines and glycols in indoor air from ENDSs (78). A study on indoor air quality and outdoor exposures of ENDS emission demonstrated that significant amounts of 1,2-propanediol, glycerine, and nicotine were found in the gas phase in the indoor air that could potentially pose as a health risk for nonusers (79). The second-hand adverse health effects of traditional cigarettes is well established but little is known about ENDS. While some recent studies are addressing this growing concern to help reduce the gap in knowledge, more research needs to be done from epidemiological and regulatory standpoints to ensure the short- or long-term negative health effects of unregulated ENDS use are not overlooked $(80,81)$. Therefore, additional comprehensive research on the implications of potential second-hand and third-hand exposure to non-users of ENDS is warranted, that allows informative communication of its potential harmful effects to the public.

\section{DISCUSSION}

Due to their relatively short-term existence on the market and limited history of use, little has been studied regarding the long-term adverse effects of ENDS. This review has identified that ENDS use remains largely undefined and unregulated, including an increasingly high prevalence of use among youth. Currently, no regulation requires ENDS manufacturers to include a warning-label on packaging regarding risks of thermal or blast injury. The public health community is divided on the health impact of vaping and smoking. Over the past few years, ENDS have become a preferred alternative to conventional cigarettes (82). Several studies have suggested that ENDS may have the potential to aid smoking cessation, but the health risk of ENDS are less known or studied. With ENDS being a recently developed product with far fewer users, limited data are available on long-term health effects of ENDS compared to those of conventional smoking. While the majority of studies on ENDS have focused on the health effects, risks of device explosion which cause thermal or chemical burns and blast injuries are now coming to the forefront $(12,83)$.

Based on analysis of the possibility of nicotine dependency and the renormalization of public smoking, the Canadian Pediatric Society has provided recommendations to help govern the ENDS industry. Some of these regulatory steps included the addition of package warnings of potential harmful effects, providing a list of ingredients and measure of the nicotine concentration on the product, banning ENDS advertisements and marketing, restricting internet sales of ENDS, and enforcing an age limit for the purchase, possession and use of the ENDS (84). In addition, the European Union (EU), Brazil and Singapore have placed restrictions on the sale, marketing, and promotion of ENDS flavors (85).

In recent years, due to emerging health hazards found to be associated with ENDS use, the FDA extended its regulatory authority on ENDS manufacture, including its components and other marketing aspects such as import, packaging, labeling, advertising, promotion, sale, and distribution. The FDA awareness of recent reports of battery-related accidents has led to interest in gaining knowledge about ENDS battery safety hazards and controls, including internal and external batteryrelated factors, specifications, safety, and design parameters of the ENDS apparatus. Variability of nicotine levels in the e-liquid, especially when higher than the labeled amount, further demonstrates the 
need for regulation of ENDS use. In response to the FDA's concerns about addiction and health effects of ENDS, the ENDS manufacturers are now required to label the device with the warning of nicotine addiction.

Most importantly, ENDS product manufacturers, importers, distributers, wholesalers, and retailers must warn the end-users through proper product labeling or manuals about the risk of overheating, fire, explosion, or other modes of failure (Figure 5). Further safety information should be provided detailing the proper battery charging processes, frequency of replacement, and maximum lifetime of the battery. Research and education are warranted regarding users' understanding of ENDS safety handling and storage (5). FDA could enforce checks through the pre-market approval process required for most FDA-regulated products, to make the ENDS consumers aware of its health threats. This would compel ENDS manufacturers to make approved design changes rendering their new or modified products safer - and eliminating the wait for manufacturers to voluntarily develop standards. While such an effort will take time and resources to create the necessary regulations and standardize the safety of ENDS devices, awareness of the problem is the essential first step in this direction. Furthermore, additional rigorous scientific studies are needed to fully characterize the addiction, toxicity, injury, and health risks associated with acute and chronic use of ENDs. The health effects of using ENDS are not well understood, and there is not enough data to prove that ENDS is less harmful than conventional cigarettes. If ENDS are used as a nicotine-free source for smoking cessation, then they could replace conventional smoking and reduce the burden of many smoking related diseases.

\section{CONCLUSION}

The increasing use and popularity of ENDS has raised concern from the public, FDA, and healthcare professionals. Numerous studies have reported accidents related to the explosion of ENDS, resulting in internal and external injuries to users. The adverse health effects from nicotine and risk of addiction in youth have also been reported from ENDS adolescent users. Various studies have identified potential risks including heavy metal leaching, toxicity from the e-liquid ingredient by-products and flavors, second-hand or third-hand exposure, and in vitro evidence of lung inflammation and cardiovascular problems. Taken together, the negative physiological effects of ENDS not only impact the user but present a challenge for the health care system to address. The number of alarming incidents of physical injuries, and unknown shortand long-term harmful effects of ENDS use call for better federal regulations to preserve the health of the public.

ACKNOWLEDGEMENTS. The authors thank Ms. Ruby J. Siegel for critical reading of the manuscript.

RESEARCH FUNDING. This research did not receive any specific grant from funding agencies in the public, commercial, or not-for-profit sectors.

AUTHORS' CONTRIBUTIONS. All the authors have accepted responsibility for the entire content of this manuscript and approved its submission.

COMPETING INTERESTS. The authors declare that they have no competing interests.

INFORMED CONSENT. Informed consent was not needed for this study.

ETHICAL APPROVAL. The conducted research is not related to either human or animal use.

\section{REFERENCES}

1. Brown CJ, Cheng JM. Electronic cigarettes: product characterisation and design considerations. Tob Control. 2014;23 Suppl 2:ii4-10.

2. Vyncke T, De Wolf E, Hoeksema H, Verbelen J, De Coninck P, Buncamper M, et al. Injuries associated with electronic nicotine delivery systems: A systematic review. J Trauma Acute Care Surg. 2020;89(4):783-91.

3. Brownson EG, Thompson CM, Goldsberry S, Chong HJ, Friedrich JB, Pham TN, et al. Explosion Injuries from E-Cigarettes. N Engl J Med. 2016;375(14):1400-2.

4. Hou J, Lu L, Wang L, Ohma A, Ren D, Feng $\mathrm{X}$, et al. Thermal runaway of Lithium-ion batteries employing LiN(SO. Nat Commun. 2020;11(1):5100.

5. Battery Safety Concerns in Electronic Nicotine Delivery Systems (ENDS) Public Workshop - April 2017, (2017). 


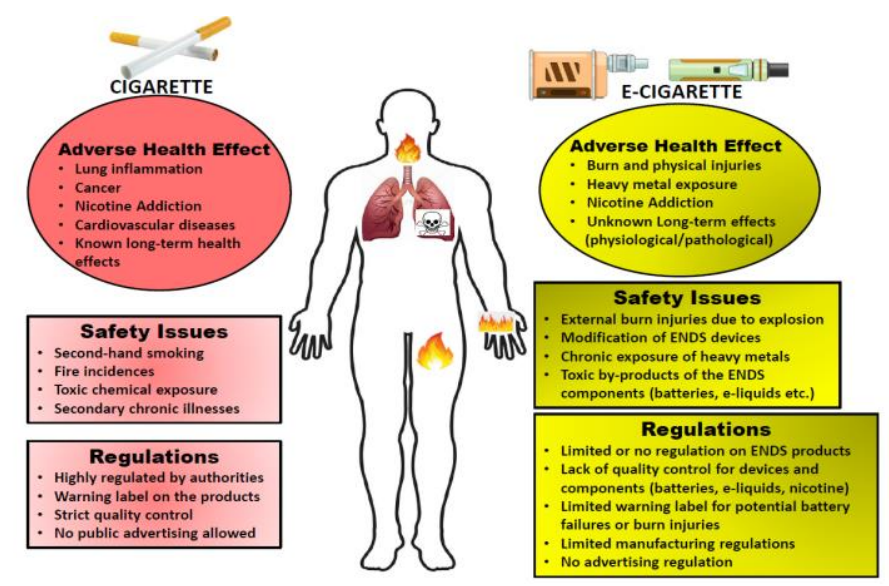

Figure 5. Comparison of adverse health effect, safety issue and regulation of cigarette with e-cigarette.

6. Chen S, Wang Z, Yan W. Identification and characteristic analysis of powder ejected from a lithium ion battery during thermal runaway at elevated temperatures. J Hazard Mater. 2020;400:123169.

7. Khairudin MN, Mohd Zahidin AZ, Bastion ML. Front to back ocular injury from a vapingrelated explosion. BMJ Case Rep. 2016;2016.

8. Welter P, Ryu SM, Pierson T, Menke H. [Danger in the pocket: explosive e-cigarette]. Handchir Mikrochir Plast Chir. 2020;52(6):490-6.

9. Beining T, Thogmartin JR, Kurz W. Projectile Wound to Head from Modified Electronic Cigarette Explosion. J Forensic Sci. 2020;65(4):1365-7.

10. Rogér JM, Abayon M, Elad S, Kolokythas A. Oral Trauma and Tooth Avulsion Following Explosion of E-Cigarette. J Oral Maxillofac Surg. 2016;74(6):1181-5.

11. Bohr S, Almarzouqi F, Pallua N. Extensive burn injury caused by fundamental electronic cigarette design flaw. Ann Burns Fire Disasters. 2016;29(3):231-3.

12. Nicoll KJ, Rose AM, Khan MA, Quaba O, Lowrie AG. Thigh burns from exploding ecigarette lithium ion batteries: First case series. Burns. 2016;42(4):e42-6.

13. Clare RA, Krenzelok EP. Chemical burns secondary to elemental metal exposure: two case reports. Am J Emerg Med. 1988;6(4):355-7.

14. Maraqa T, Mohamed MAT, Salib M, Morris S, Mercer L, Sachwani-Daswani GR. Too Hot for Your Pocket! Burns From E-Cigarette Lithium Battery Explosions: A Case Series. J Burn Care Res. 2018;39(6):1043-7.

15. Norii $\mathrm{T}$, Plate A. Electronic Cigarette Explosion Resulting in a C1 and C2 Fracture: A Case Report. J Emerg Med. 2017;52(1):868.

16. Paley GL, Echalier E, Eck TW, Hong AR, Farooq AV, Gregory DG, et al. Corneoscleral Laceration and Ocular Burns Caused by Electronic Cigarette Explosions. Cornea. 2016;35(7):1015-8.

17. Foran I, Oak NR, Meunier MJ. High-Pressure Injection Injury Caused by Electronic Cigarette Explosion: A Case Report. JBJS Case Connect. 2017;7(2):e36.

18. Serror K, Chaouat M, Legrand MM, Depret F, Haddad J, Malca N, et al. Burns caused by electronic vaping devices (e-cigarettes): $\mathrm{A}$ new classification proposal based on mechanisms. Burns. 2018;44(3):544-8.

19. Brooks JK, Kleinman JW, Brooks JB, Reynolds MA. Electronic cigarette explosion associated with extensive intraoral injuries. Dent Traumatol. 2017;33(2):149-52.

20. Patterson SB, Beckett AR, Lintner A, Leahey C, Greer A, Brevard SB, et al. A Novel Classification System for Injuries After Electronic Cigarette Explosions. J Burn Care Res. 2017;38(1):e95-e100.

21. Quiroga L, Asif M, Lagziel T, Bhat D, Caffrey J. E-Cigarette Battery Explosions: Review of the Acute Management of the Burns and the 
Impact on Our Population. Cureus. 2019;11(8):e5355.

22. Jones CD, Ho W, Gunn E, Widdowson D, Bahia H. E-cigarette burn injuries: Comprehensive review and management guidelines proposal. Burns. 2019;45(4):76371.

23. McKenna Jr. L. Electronic Cigarette Fires and Explosions in the United States 2009-2016. U.S. Fire Administration, FEMA, U.S. Department of Homeland Security; 2017.

24. Kite AC, Le BQ, Cumpston KL, Hieger MA, Feldman MJ, Pozez AL. Blast Injuries Caused by Vape Devices: 2 Case Reports. Ann Plast Surg. 2016;77(6):620-2.

25. Food and Drug Administration HHS. Deeming Tobacco Products To Be Subject to the Federal Food, Drug, and Cosmetic Act, as Amended by the Family Smoking Prevention and Tobacco Control Act; Restrictions on the Sale and Distribution of Tobacco Products and Required Warning Statements for Tobacco Products. Final rule. Fed Regist. 2016;81(90):28973-9106.

26. Gray N, Halstead M, Gonzalez-Jimenez N, Valentin-Blasini L, Watson C, Pappas RS. Analysis of Toxic Metals in Liquid from Electronic Cigarettes. Int J Environ Res Public Health. 2019;16(22).

27. Clapp PW, Jaspers I. Electronic Cigarettes: Their Constituents and Potential Links to Asthma. Curr Allergy Asthma Rep. 2017;17(11):79.

28. Wang P, Chen W, Liao J, Matsuo T, Ito K, Fowles J, et al. A Device-Independent Evaluation of Carbonyl Emissions from Heated Electronic Cigarette Solvents. PLoS One. 2017;12(1):e0169811.

29. Salamanca JC, Meehan-Atrash J, Vreeke S, Escobedo JO, Peyton DH, Strongin RM. Ecigarettes can emit formaldehyde at high levels under conditions that have been reported to be non-averse to users. Sci Rep. 2018;8(1):7559.

30. Farsalinos KE, Voudris V, Spyrou A, Poulas K. E-cigarettes emit very high formaldehyde levels only in conditions that are aversive to users: A replication study under verified realistic use conditions. Food Chem Toxicol. 2017;109(Pt 1):90-4.

31. Ordonez JE, Kleinschmidt KC, Forrester MB. Electronic cigarette exposures reported to
Texas poison centers. Nicotine Tob Res. 2015;17(2):209-11.

32. Kozlovich S, Chen G, Watson CJW, Blot WJ, Lazarus P. Role of 1- and d-Menthol in the Glucuronidation and Detoxification of the Major Lung Carcinogen, NNAL. Drug Metab Dispos. 2019;47(12):1388-96.

33. Muscat JE, Chen G, Knipe A, Stellman SD, Lazarus P, Richie JP. Effects of menthol on tobacco smoke exposure, nicotine dependence, and NNAL glucuronidation. Cancer Epidemiol Biomarkers Prev. 2009;18(1):35-41.

34. Lucas JH, Muthumalage T, Wang Q, Friedman MR, Friedman AE, Rahman I. ELiquid Containing a Mixture of Coconut, Vanilla, and Cookie Flavors Causes Cellular Senescence and Dysregulated Repair in Pulmonary Fibroblasts: Implications on Premature Aging. Front Physiol. 2020;11:924.

35. Abouassali O, Chang M, Chidipi B, Martinez JL, Reiser M, Kanithi M, et al. In vitro and in vivo cardiac toxicity of flavored electronic nicotine delivery systems. Am J Physiol Heart Circ Physiol. 2021;320(1):H133-H43.

36. National Academies of Sciences Eg, and Medicine, Division HaM, Practice BoPHaPH, Systems CotRotHEoEND. Public Health Consequences of E-Cigarettes. 2018.

37. Lerner CA, Rutagarama P, Ahmad T, Sundar IK, Elder A, Rahman I. Electronic cigarette aerosols and copper nanoparticles induce mitochondrial stress and promote DNA fragmentation in lung fibroblasts. Biochem Biophys Res Commun. 2016;477(4):620-5.

38. Durrani K, El Din SA, Sun Y, Rule AM, Bressler J. Ethyl maltol enhances copper mediated cytotoxicity in lung epithelial cells. Toxicol Appl Pharmacol. 2021;410:115354.

39. Kankanamage RNT, Ghosh AB, Jiang D, Gkika K, Keyes T, Achola LA, et al. Metabolites of Tobacco- and E-CigaretteRelated Nitrosamines Can Drive $\mathrm{Cu}$. Chem Res Toxicol. 2020;33(8):2072-86.

40. Sharma A, Lee J, Fonseca AG, Moshensky A, Kothari T, Sayed IM, et al. E-cigarettes compromise the gut barrier and trigger inflammation. iScience. 2021;24(2):102035.

41. Aherrera A, Olmedo P, Grau-Perez M, Tanda $\mathrm{S}$, Goessler W, Jarmul S, et al. The association of e-cigarette use with exposure to nickel and chromium: A preliminary study of non- 
invasive biomarkers. Environ Res. 2017;159:313-20.

42. Kumetz EA, Hurst ND, Cudnik RJ, Rudinsky SL. Electronic cigarette explosion injuries. Am J Emerg Med. 2016;34(11):2252 e1- e3.

43. Durmowicz EL. The impact of electronic cigarettes on the paediatric population. Tob Control. 2014;23 Suppl 2:ii41-6.

44. Eadie D, Stead M, MacKintosh AM, MacDonald L, Purves R, Pearce J, et al. Ecigarette marketing in UK stores: an observational audit and retailers' views. BMJ Open. 2015;5(9):e008547.

45. Jackson R, Huskey M, Brown S. Labelling accuracy in low nicotine e-cigarette liquids from a sampling of US manufacturers. Int $\mathbf{J}$ Pharm Pract. 2020;28(3):290-4.

46. Wasowicz A, Feleszko W, Goniewicz ML. ECigarette use among children and young people: the need for regulation. Expert Rev Respir Med. 2015;9(5):507-9.

47. England LJ, Bunnell RE, Pechacek TF, Tong VT, McAfee TA. Nicotine and the Developing Human: A Neglected Element in the Electronic Cigarette Debate. Am J Prev Med. 2015;49(2):286-93.

48. Buettner-Schmidt K, Miller DR, Balasubramanian N. Electronic Cigarette Refill Liquids: Child-Resistant Packaging, Nicotine Content, and Sales to Minors. J Pediatr Nurs. 2016;31(4):373-9.

49. Payne JD, Michaels D, Orellana-Barrios M, Nugent K. Electronic Cigarette Toxicity. J Prim Care Community Health. 2017;8(2):1002.

50. Wang MP, Ho SY, Leung LT, Lam TH. Electronic Cigarette Use and Respiratory Symptoms in Chinese Adolescents in Hong Kong. JAMA Pediatr. 2016;170(1):89-91.

51. Cho JH. The association between electroniccigarette use and self-reported oral symptoms including cracked or broken teeth and tongue and/or inside-cheek pain among adolescents: A cross-sectional study. PLoS One. 2017;12(7):e0180506.

52. Hanewinkel R, Isensee B. Risk factors for ecigarette, conventional cigarette, and dual use in German adolescents: a cohort study. Prev Med. 2015;74:59-62.

53. Forrester MB. Pediatric Exposures to Electronic Cigarettes Reported to Texas Poison Centers. J Emerg Med. 2015;49(2):136-42.
54. Menakuru S, Inzamam Ali M. Beliefs and reality of e-cigarette smoking. BMJ Case Rep. 2018;2018.

55. Case KR, Mantey DS, Creamer MR, Harrell MB, Kelder SH, Perry CL. E-cigarettespecific symptoms of nicotine dependence among Texas adolescents. Addict Behav. 2018;84:57-61.

56. Morean ME, Krishnan-Sarin S, S SOM. Assessing nicotine dependence in adolescent E-cigarette users: The 4-item Patient-Reported Outcomes Measurement Information System (PROMIS) Nicotine Dependence Item Bank for electronic cigarettes. Drug Alcohol Depend. 2018;188:60-3.

57. Cavazos-Rehg P, Li X, Kasson E, Kaiser N, Borodovsky J, Grucza RA. Investigating the role of familial and peer-related factors on electronic nicotine delivery systems (ENDS) use among U.S. adolescents. J Adolesc. 2021;87:98-105.

58. Marsot A, Simon N. Nicotine and Cotinine Levels With Electronic Cigarette: A Review. Int J Toxicol. 2016;35(2):179-85.

59. Morean ME, Krishnan-Sarin S, Sussman S, Foulds J, Fishbein $\mathrm{H}$, Grana $\mathrm{R}$, et al. Psychometric Evaluation of the E-cigarette Dependence Scale. Nicotine Tob Res. 2019;21(11):1556-64.

60. Dawkins L, Corcoran O. Acute electronic cigarette use: nicotine delivery and subjective effects in regular users. Psychopharmacology (Berl). 2014;231(2):401-7.

61. Eissenberg T. Electronic nicotine delivery devices: ineffective nicotine delivery and craving suppression after acute administration. Tob Control. 2010;19(1):87-8.

62. Flouris AD, Chorti MS, Poulianiti KP, Jamurtas AZ, Kostikas K, Tzatzarakis MN, et al. Acute impact of active and passive electronic cigarette smoking on serum cotinine and lung function. Inhal Toxicol. 2013;25(2):91-101.

63. Etter JF, Bullen C. Saliva cotinine levels in users of electronic cigarettes. Eur Respir J. 2011;38(5):1219-20.

64. Vansickel AR, Cobb CO, Weaver MF, Eissenberg TE. A clinical laboratory model for evaluating the acute effects of electronic "cigarettes": nicotine delivery profile and cardiovascular and subjective effects. Cancer Epidemiol Biomarkers Prev. 2010;19(8):1945-53. 
65. Bullen C, McRobbie H, Thornley S, Glover M, Lin R, Laugesen M. Effect of an electronic nicotine delivery device (e cigarette) on desire to smoke and withdrawal, user preferences and nicotine delivery: randomised cross-over trial. Tob Control. 2010;19(2):98-103.

66. Vansickel AR, Eissenberg T. Electronic cigarettes: effective nicotine delivery after acute administration. Nicotine Tob Res. 2013;15(1):267-70.

67. Perkins KA, Jao NC, Karelitz JL. Consistency of daily cigarette smoking amount in dependent adults. Psychol Addict Behav. 2013;27(3):723-9.

68. Augustson E, Marcus S. Use of the current population survey to characterize subpopulations of continued smokers: a national perspective on the "hardcore" smoker phenomenon. Nicotine Tob Res. 2004;6(4):621-9.

69. Shiffman S. How many cigarettes did you smoke? Assessing cigarette consumption by global report, Time-Line Follow-Back, and ecological momentary assessment. Health Psychol. 2009;28(5):519-26.

70. Klesges RC, Debon M, Ray JW. Are selfreports of smoking rate biased? Evidence from the Second National Health and Nutrition Examination Survey. J Clin Epidemiol. 1995;48(10):1225-33.

71. Huey S, Granitto M. Escape the vape: Health hazards of the latest nicotine craze. Nursing. 2017;47(1):46-51.

72. Hartmann-Boyce J, McRobbie H, Lindson N, Bullen C, Begh R, Theodoulou A, et al. Electronic cigarettes for smoking cessation. Cochrane Database Syst Rev. 2020;10:CD010216.

73. Polosa R, Morjaria JB, Caponnetto P, Caruso $\mathrm{M}$, Campagna $\mathrm{D}$, Amaradio $\mathrm{MD}$, et al. Persisting long term benefits of smoking abstinence and reduction in asthmatic smokers who have switched to electronic cigarettes. Discov Med. 2016;21(114):99-108.

74. Polosa R, Morjaria JB, Prosperini U, Russo C, Pennisi A, Puleo R, et al. Health effects in COPD smokers who switch to electronic cigarettes: a retrospective-prospective 3-year follow-up. Int J Chron Obstruct Pulmon Dis. 2018;13:2533-42.

75. George J, Hussain M, Vadiveloo T, Ireland S, Hopkinson P, Struthers AD, et al. Cardiovascular Effects of Switching
From Tobacco Cigarettes to Electronic Cigarettes. J Am Coll Cardiol. 2019;74(25):3112-20.

76. Hajek P, Phillips-Waller A, Przulj D, Pesola F, Myers Smith K, Bisal N, et al. A Randomized Trial of E-Cigarettes versus NicotineReplacement Therapy. N Engl J Med. 2019;380(7):629-37.

77. Almeida-da-Silva CLC, Matshik Dakafay H, O'Brien K, Montierth D, Xiao N, Ojcius DM. Effects of electronic cigarette aerosol exposure on oral and systemic health. Biomed J. 2020.

78. Brandon TH, Goniewicz ML, Hanna NH, Hatsukami DK, Herbst RS, Hobin JA, et al. Electronic nicotine delivery systems: a policy statement from the American Association for Cancer Research and the American Society of Clinical Oncology. Clin Cancer Res. 2015;21(3):514-25.

79. Schober W, Szendrei K, Matzen W, OsianderFuchs H, Heitmann D, Schettgen T, et al. Use of electronic cigarettes (e-cigarettes) impairs indoor air quality and increases FeNO levels of e-cigarette consumers. Int $\mathrm{J}$ Hyg Environ Health. 2014;217(6):628-37.

80. Fried ND, Gardner JD. Heat-not-burn tobacco products: an emerging threat to cardiovascular health. Am J Physiol Heart Circ Physiol. 2020;319(6):H1234-H9.

81. Qasim H, Alarabi AB, Alzoubi KH, Karim ZA, Alshbool FZ, Khasawneh FT. The effects of hookah/waterpipe smoking on general health and the cardiovascular system. Environ Health Prev Med. 2019;24(1):58.

82. Caponnetto P, Campagna D, Papale G, Russo C, Polosa R. The emerging phenomenon of electronic cigarettes. Expert Rev Respir Med. 2012;6(1):63-74.

83. Treitl D, Solomon R, Davare DL, Sanchez R, Kiffin C. Full and Partial Thickness Burns from Spontaneous Combustion of E-Cigarette Lithium-Ion Batteries with Review of Literature. J Emerg Med. 2017;53(1):121-5.

84. Stanwick R. E-cigarettes: Are we renormalizing public smoking? Reversing five decades of tobacco control and revitalizing nicotine dependency in children and youth in Canada. Paediatr Child Health. 2015;20(2):101-5.

85. Duderstadt KG. E-Cigarettes: Youth and Trends in Vaping. J Pediatr Health Care. 2015;29(6):555-7. 